\title{
Distributed contact flip chip InGaN/GaN blue LED; comparison with conventional LEDs
}

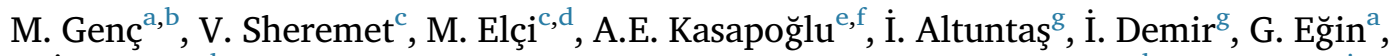 \\ S. İslamoğlu ${ }^{\mathrm{h}}$, Emre Gürr $^{\mathrm{e}, *}$, N. Muzafferoğlu ${ }^{\mathrm{a}}, \mathrm{S}$. Elagöz ${ }^{\mathrm{g}}$, O. Gülseren ${ }^{\mathrm{h}}, \mathrm{A}$. Aydınlı ${ }^{\mathrm{i}, * *}$ \\ ${ }^{a}$ Ermaksan A.Ş., Optoelectronic R\&D Center, 16065, Bursa, Turkey \\ ${ }^{\mathrm{b}}$ Bursa Technical University, Department of Material Science and Engineering, 16310, Bursa, Turkey \\ ${ }^{\mathrm{c}}$ Bilkent University, Advanced Research Laboratories, 06800, Ankara, Turkey \\ ${ }^{\mathrm{d}}$ Institute of Applied Mathematics, Middle East Technical University, Ankara, 06800, Turkey \\ e Atatürk University, Department of Physics, 25240, Erzurum, Turkey \\ ${ }^{\mathrm{f}}$ Atatïrk University, East Anatolia High Technology Application and Research Center, 25240, Erzurum, Turkey \\ ${ }^{\mathrm{g}}$ Cumhuriyet University, Nanotechnology Research Center, 58140, Sivas, Turkey \\ ${ }^{\mathrm{h}}$ Bilken University, Department of Physics, 06800, Ankara, Turkey \\ ${ }^{\mathrm{i}}$ Uludağ University, Electrical and Electronics Engineering Department, 16059, Bursa, Turkey
}

\section{A R T I C L E I N F O}

\section{Keywords:}

Light emitting diodes

Luminescence

LED performance

Current distribution

\begin{abstract}
A B S T R A C T
This paper presents high performance, GaN/InGaN-based light emitting diodes (LEDs) in three different device configurations, namely Top Emitting (TE) LED, conventional Flip Chip (FC) and Distributed Contact (DC) FC. Series resistances as low as $1.1 \Omega$ have been obtained from FC device configurations with a back reflecting ohmic contact of Ni/Au/RTA/Ni/Ag metal stack. A small shift has been observed between electroluminescence (EL) emissions of TE LED and the FC LEDs. In addition, FWHM value of the EL emission of DCFC LED has shown the minimum value of $160 \mathrm{meV}(26.9 \mathrm{~nm})$. Furthermore, DCFC LED configuration has shown the highest quantum efficiency and power output, with $330 \mathrm{~mW}$ at $500 \mathrm{~mA}$ current injection, compared to that of traditional wire bonded TE LEDs and the conventional FC LEDs.
\end{abstract}

\section{Introduction}

$\mathrm{In}_{\mathrm{x}} \mathrm{Ga}_{1-\mathrm{x}} \mathrm{N}$ ternary compound semiconductor spans the entire visible spectrum making it a very important material system for many different solid state device applications [1]. Potential use in solid state lighting makes this semiconductor system invaluable and especially indispensable in the shorter wavelength regime of the visible spectrum for optoelectronic applications such as laser diodes (LDs) and light emitting diodes (LEDs) [2]. An important demand on these device applications is high light extraction efficiency (LEE) which is vital, in particular, for lightening applications. Many different epitaxial layer designs as well as the different device configurations have been employed to increase LED LEE [3-6]. One of the most common InGaN/GaN LED device configurations is the conventional top emitting LEDs (TE LEDs) which include a variety of top current spreading layers, such as thin $\mathrm{Ni} / \mathrm{Au}$ metallization, ITO, AZO and etc., and interdigitated metal contact based structures [7]. In the conventional TE LEDs, LEE is significantly decreased due to the absorption of the photons generated within the device by the metal top contacts and/or the bonding pads on the p-type GaN layer. Furthermore, high power input causes degradation of the optical performance, reliability and lifetime

\footnotetext{
${ }^{*}$ Corresponding author.

** Corresponding author.

E-mail addresses: emregur@atauni.edu.tr (E. Gür), atillaaydinli@uludag.edu.tr (A. Aydınlı).
} 
of the optoelectronic device due to the detrimental thermal loading effects. In order to mitigate the thermal problem and increase current spreading, flip chip (FC) LED configuration has been commonly adopted [8]. Since the chip is flipped over and mounted on a heat sink sub-mount, issues such as high ohmic contact resistance and absorption of light due to the top metallic fingers on p-GaN are mitigated. FC LEDs also gain an extra advantage from the thick backside reflector which increases the efficiency. In addition to the conventional FC LED device structures, there are also thin film FC LED device configurations which require elimination of the sapphire substrate and the surface roughening of the n-type GaN layer [9]. These extra processing steps bring out extra device costs which might not be applicable especially for the mid-power LEDs.

In the present study, performance of the Distributed Contact Flip Chip LEDs (DCFC) device configuration is compared with the conventional TE LED and conventional FC LED in terms of the total output power, external quantum efficiency (EQE) and the currentvoltage $(\mathrm{I}-\mathrm{V})$ characteristics. Despite commercial implementation little is available on DCFC type LEDs, recently, there have been increasing trend in terms of the researches in these LED configurations [10-13]. It has been commonly shown that DCFC LEDs has indicated better device performances compared to the traditional TE LEDs.

\section{Experimental}

The epitaxial structure of InGaN/GaN Multiple Quantum Well (MQW) based LED structures was grown by metalorganic chemical vapor deposition (MOCVD) technique on $3300 \mathrm{~nm} \mathrm{n-GaN} \mathrm{layer,} \mathrm{itself} \mathrm{grown} \mathrm{on} \mathrm{patterned} \mathrm{sapphire} \mathrm{substrates.} \mathrm{Active} \mathrm{region} \mathrm{consists}$ of $390 \mathrm{~nm}$ thick InGaN/GaN MQW. Electron and hole blocking layer of $20 \mathrm{~nm} \mathrm{AlGaN} \mathrm{both} \mathrm{top} \mathrm{and} \mathrm{bottom} \mathrm{of} \mathrm{the} \mathrm{active} \mathrm{region} \mathrm{was}$ grown. $20 \mathrm{~nm}$ InGaN layer was grown on top of the structure to obtain better ohmic contact to p-GaN layer which is $280 \mathrm{~nm}$ thick. Mesas were formed by Inductively Coupled Plasma (ICP) etching $\left(\mathrm{BCl}_{3}: \mathrm{Cl}_{2}\right)$. Ohmic contacts to n-GaN for all the three different device configurations were formed using a $\mathrm{Ti}(30 \mathrm{~nm}) / \mathrm{Al}(50 \mathrm{~nm}) / \mathrm{TiB}_{2}(50 \mathrm{~nm}) / \mathrm{Au}(70 \mathrm{~nm})$ metal stack which underwent rapid thermal annealing (RTA) in $\mathrm{N}_{2}$ at $\mathrm{T}=850^{\circ} \mathrm{C}$ for $1 \mathrm{~min}$. Ohmic contact to $\mathrm{p}-\mathrm{GaN}$ in TE LED device was $\mathrm{Ni}(5 \mathrm{~nm}) / \mathrm{Au}(5 \mathrm{~nm}) / \mathrm{ITO}(120 \mathrm{~nm})$ used with $\mathrm{Ti}(30 \mathrm{~nm}) / \mathrm{Au}(100 \mathrm{~nm})$ contact pads, while it is $\mathrm{Ni}(7.5 \mathrm{~nm}) / \mathrm{Au}(7.5 \mathrm{~nm}) / \mathrm{RTA} / \mathrm{Ni}(5 \mathrm{~nm}) / \mathrm{Ag}(160 \mathrm{~nm})$ metal stack with $\mathrm{Cr}$ $(30 \mathrm{~nm}) / \mathrm{Au}(170 \mathrm{~nm})$ contact pads in both FC LED devices. The passivation between the p-GaN and n-GaN ohmic contacts was made by $250 \mathrm{~nm}$ thick $\mathrm{SiO}_{2}$ grown by plasma enhanced chemical vapor deposition immediately after ICP mesa-etching in all the three different device configurations. All n-contact holes shorted to each other using Ti/Au metals for the case of DCFC LED.

Fig. 1, shows the sketch of the DCFC LED after all lithography processes. After etching the holes down to the n-GaN layer, the walls of the holes were isolated. The increased p-contact area and the distributed nature of n-contacts with these holes is intended to assure lower series resistance, better current distribution and higher efficiency. Final contact metal lithography separates n- and pcontacts for soldering to the submount isolating them from each other with PECVD grown $\mathrm{SiO}_{2}$.

Electrical properties of fabricated LED dies were measured using a Keithley 2430 source and measurement unit of a HP 4142B semiconductor parametric analyzer. Photoluminescence (PL) measurements were performed by $325 \mathrm{~nm}, 60 \mathrm{~mW} \mathrm{He}-\mathrm{Cd}$ laser, which is focused on the $10 \times$ objective lens. The signal was detected by the PMT detector attached to the $750 \mathrm{~mm}$ Czerny-Turner type monochromator with $1200 \mathrm{gr} / \mathrm{mm}$ ruled grating. A lock-in amplifier was used to analyze the data. Slit width was $10 \mu \mathrm{m}$ which results in an overall resolution of about $0.35 \AA$. Electroluminescence (EL) measurements were studied by Instrument Systems spectrometer CAS 140CT. Packaged TE LED, FC LED and DCFC LED samples were placed in the $150 \mathrm{~mm}$ integrating sphere to obtain total output power of the chips. Ohmic contact resistivity values were determined by using transmission line method (TLM) with linear and circular contact pad geometries. For linear TLM measurements, $75 \times 450 \mu^{2}$ contact pads were used with separations of 20 , 40, 60 , 80,100 , and $150 \mu \mathrm{m}$. For circular TLM measurements inner contact pads had diameters of 50, 80, 100, and 150 $\mu \mathrm{m}$ with constant ratio between outer and inner contact diameters equal to 2 .

\section{Results and discussions}

Fig. 2 shows low temperature PL measurement on the epi structure and EL measurements of three different LED configurations under $20 \mathrm{~mA}$ injection current conditions. As seen from the PL measurements, there are multiple peaks appearing at energies of $3.47 \mathrm{eV}, 3.28 \mathrm{eV}, 3.20 \mathrm{eV}$ and $2.76 \mathrm{eV}$ and $2.67 \mathrm{eV}$. The emission observed at the $3.47 \mathrm{eV}$ belongs to the GaN band edge emission [14],

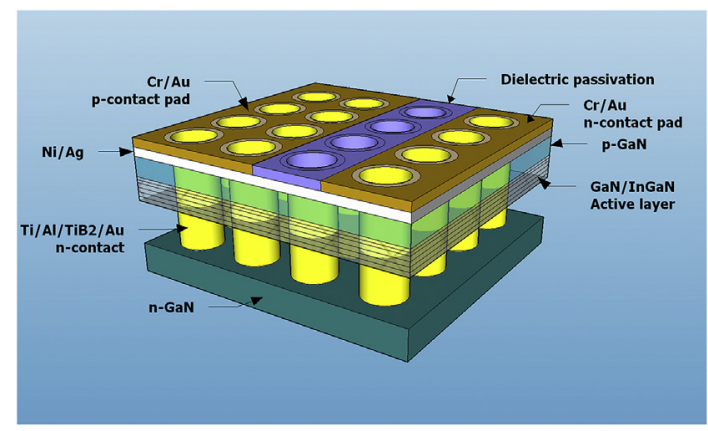

Fig. 1. Sketch of the DCFC LED after lithography. 


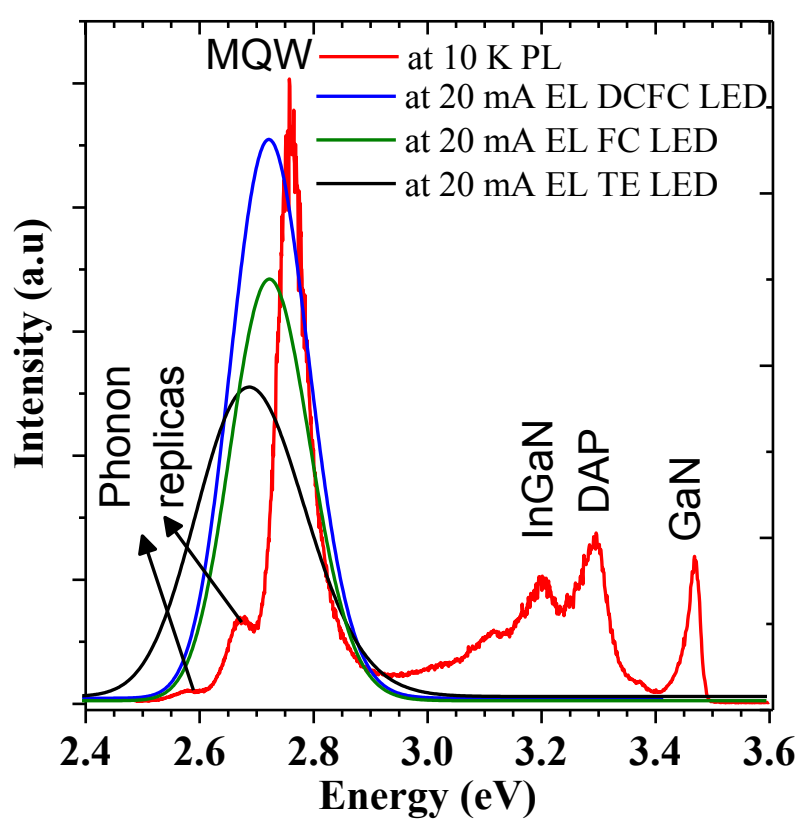

Fig. 2. PL of the epitaxial structure and EL of the three different LEDs under $20 \mathrm{~mA}$ current injection conditions.

while the emission at $3.28 \mathrm{eV}$ is commonly attributed to donor-acceptor pair (DAP) transition in the p-GaN layer [15]. Band edge emission of the top $20 \mathrm{~nm}$ thick InGaN layer has been observed at energy of $3.20 \mathrm{eV}$. The emission of MQW is seen at $2.76 \mathrm{eV}$ which has a full width at half maximum (FWHM) value of $63.0 \mathrm{meV}(10.3 \mathrm{~nm})$. At lower energy side of this emission, there are small peaks observed at $2.67 \mathrm{eV}$ and $2.58 \mathrm{eV}$, which is lower than $90 \mathrm{meV}$ from each other's. As the LO-phonon energy of $90 \mathrm{meV}$ in $\mathrm{GaN}$ is considered [14], these peaks might be attributed to the phonon replicas of the quantum well. On the other hand, EL emissions observed are at $2.73 \mathrm{eV}$ for both FC LEDs and $2.69 \mathrm{eV}$ for TE LED under $20 \mathrm{~mA}$ current injection conditions, which is at a lower energy compared to that of PL emission energy. The observed energy difference might be due to the changes in polarization formed in the QWs when the excitation method is changed [16]. Apparently, excitation with UV laser light to induce photoluminescence has no impact on the intrinsically formed polarization in the QWs. However, the electric field due to applied voltage during EL measurements result in changes in the electric field distribution in the QWs. This changes the conduction and valance band bending which results in the shift of the emission energy. It is well known that EL emission shift to lower energies requires larger bending in the bands. This is expected in the Ga-polar InGaN/GaN LEDs [16].

FWHM values of the EL emissions shown in Fig. 2 are $222 \mathrm{meV}(38 \mathrm{~nm}$ ) for TE LED, $170 \mathrm{meV}$ (29.3 nm) for FC LED and $160 \mathrm{meV}$ $(26.9 \mathrm{~nm})$ for DCFC LED. It is clear that there is a shift in energy as well as variations in FWHM values of the EL emissions when FC LEDs and TE LED configurations are compared. The significant difference between TE and FC LEDs suggest that the observed effect is most likely due to self-heating during the operation of TE LEDs which causes both additional broadening of $52 \mathrm{meV}$ and energy shift of $40 \mathrm{meV}$ in the emission.

Fig. 3 shows the I-V characteristics of all three different LEDs. It shows that FC LEDs and DCFC LEDs reach $350 \mathrm{~mA}$ at $3.3 \mathrm{~V}$ and $4.1 \mathrm{~V}$ bias, respectively, while this is $6.4 \mathrm{~V}$ for TE LEDs. Better values for TE LEDs exist in the literature [17] and the higher resistance of our TE devices originating from their top contact configurations can be reduced with further optimization. Series resistances as low as 1.1 $\Omega$ for FC LEDs and 2.0 $\Omega$ for DCFC LEDs and 7.0 $\Omega$ for TE LEDs have been obtained, as can be seen clearly from the slopes of the curves in Fig. 3. It is lower for FC LEDs compared with TE LED as also shown from the slope at higher voltages of the I-V curves given in Fig. 3. Although both DCFC and FC LEDs have FC device configuration, the small difference between FC and DCFC LEDs series resistances is most likely due to increased complexity of DCFC LED fabrication causing small deterioration of the ohmic contacts which can be further optimized.

Another important difference observed from the $\mathrm{I}-\mathrm{V}$ measurements is the turn-on voltages of the LEDs. The turn on voltage is $2.50 \mathrm{~V}$ for both FC LEDs, while it is $3.47 \mathrm{~V}$ for the TE LED. Eliminating heat from the active region and any leakage currents along the side walls help enhance I-V characteristics in both FC and DCFC LED device configurations. Table 1 shows ohmic contact resistivity values for the metal stacks, both on $\mathrm{n}$ - and $\mathrm{p}-\mathrm{GaN}$, measured from the TLM patterns and the series resistance values obtained from the I-V curves for the three different LEDs. Contact resistivity values on the $\mathrm{n}$ - and $\mathrm{p}$-GaN layers for the TE LEDs are shown to be almost an order of magnitude higher compared to those of FC LEDs as well as higher series resistances. While the contact resistivity on pInGaN is lowest for DCFC LED device configuration, the contact resistivity value on n-GaN is lowest for FC LED. Inset shows on wafer EL measurements of each LED. The emission in all cases are uniform and show no evidence of defects.

The relationship between output power and injected current were shown in Fig. 4 (a). It can be seen clearly that output power of DCFC LEDs is largest compared with the FC LEDs and TE LEDs despite the small disadvantage in series resistance with conventional FC LED. Total power output from the devices under $500 \mathrm{~mA}$ current injection condition are 208, 288 and $330 \mathrm{~mW}$ for TE LED, FC LED 


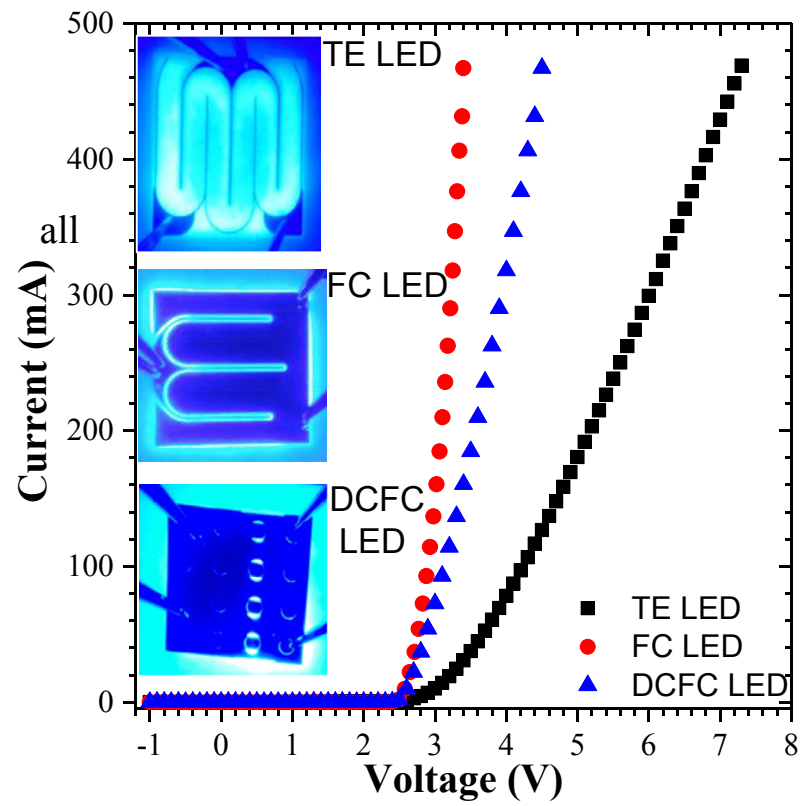

Fig. 3. I-V curve for all three different LEDs. Inset shows light emission from the three different LEDs.

Table 1

Ohmic contact resistivity and series resistance values of the three different LED.

\begin{tabular}{llll}
\hline Ohmic contacts & TE LED $\left(\mathrm{Ohm} . \mathrm{cm}^{2}\right)$ & FC LED $\left(\right.$ Ohm.cm $\left.{ }^{2}\right)$ & DCFC LED $\left(\mathrm{Ohm} . \mathrm{cm}^{2}\right)$ \\
\hline $\mathrm{Ti} / \mathrm{Al} / \mathrm{TiB}_{2} / \mathrm{Au}$ to $\mathrm{n}$-GaN & $1.7 \times 10^{-6}$ & $5.7 \times 10^{-7}$ & $7.7 \times 10^{-7}$ \\
$\mathrm{Ni} / \mathrm{Au} / \mathrm{Ni} / \mathrm{Ag}^{\mathrm{a}}$ for FC LEDs to p-InGaN & $6.5 \times 10^{-2}$ & $8.0 \times 10^{-3}$ & $4.8 \times 10^{-3}$ \\
Series resistance $(\mathrm{Ohm})$ & $7-15$ & $1.1-1.8$ & $2.0-3.5$ \\
\hline
\end{tabular}

a Ohmic contact is $\mathrm{Ni} / \mathrm{Au} / \mathrm{ITO}$ for TE LED on $\mathrm{p}-\mathrm{InGaN}$.
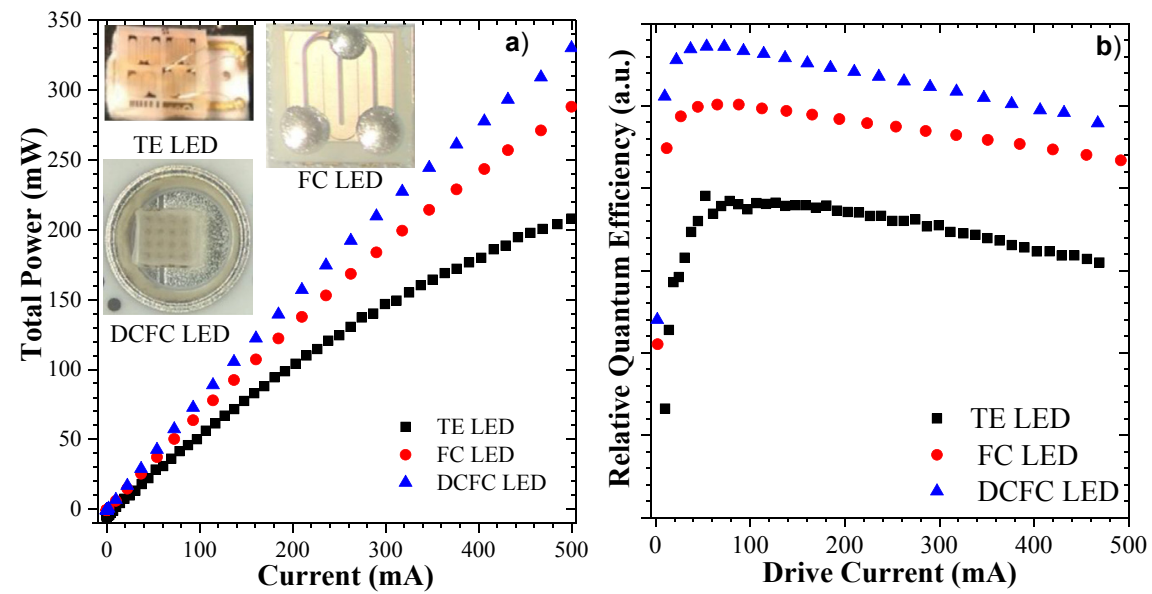

Fig. 4. a) Power output vs injected current plot for the three different LEDs. Inset shows the LED devices after packaged b) EQE values vs drive current plot for all three different LEDs.

and DCFC LED, respectively. The output power measured for DCFC LED is shown to be 58.7\% higher from TE LED and 14.6\% from the conventional FC LED. Higher performance of the FC LEDs might be due to the fact that no bonding pads or wires exist on top of the FC and DCFC LEDs. It should also be noted that since the refractive indices of sapphire substrate and GaN are 1.7 and 2.5 , respectively, photons generated in the active region should be able to reach the escape cone easier at the sapphire/air interface, compared with the GaN/air interface. Moreover, using $16 \mathrm{n}$-GaN hole contacts to distribute the current whole of the p-GaN surface contributes to light output more in the case of DCFC LEDs. It is also noticeable from the figure that the light power output starts to saturate for TE LEDs. On the other hand, the light output from DCFC LEDs increases almost linearly at least up to $500 \mathrm{~mA}$ current 
injection. Fig. 4 (b) shows the relative EQE values obtained for the LEDs for comparison. It is observed that the EQE values largest for the DCFC LED compared with the other two configurations. The larger EQE for DCFC compared to the FC LED is also significant, signaling the importance of current distribution.

In conclusion, $\mathrm{I}-\mathrm{V}$ measurement results clearly shows that different device configurations have significant impact on the electrical performance of the LEDs. The DCFC LEDs designed and fabricated in this study show significantly higher output power and external quantum efficiency under same operating conditions. Their series resistance can be optimized further down to 1 Ohm range.

\section{Acknowledgement}

We are grateful to H. Morkoç, Ü Özgür, V. Avrutin of Virginia Commonwealth University for many fruitful discussions. This work is supported by Scientific and Technical Research Council of Turkey, (TUBITAK Grant no: 113G042 and 115E109).

\section{Appendix A. Supplementary data}

Supplementary data to this article can be found online at https://doi.org/10.1016/j.spmi.2019.01.008.

\section{References}

[1] Yasushi Nanishi, Yoshiki Saito, Tomohiro Yamaguchi, RF-molecular beam epitaxy growth and properties of InN and related alloys, Jpn. J. Appl. Phys. 42 (2003) 2549.

[2] R. Stevenson, Lasers get the green light, IEEE Spectrum 47 (2010) 34.

[3] Jae-Hoon Lee, Seok-Min Hwang, Nam-Seung Kim, Jung-Hee Lee, InGaN-Based high-power flip-chip LEDs with deep-hole-patterned sapphire substrate by laser direct beam drilling, IEEE Electron. Device Lett. 31 (10) (2010) 698.

[4] C.F. Shen, S.J. Chang, W.S. Chen, T.K. Ko, C.T. Kuo, S.C. Shei, Nitride-based high-power flip-chip LED with double-side patterned sapphire substrate, IEEE Photon. Technol. Lett. 19 (10) (2007) 780.

[5] Xiao-Long Hu, Zhao-Yi Qi, Hong Wang, Xi-Chun Zhang, Performance of InGaN-based thin-film LEDs with flip-chip configuration and concavely patterned surface fabricated on electroplating metallic substrate, IEEE Photonics J. 8 (1) (2016) 1600307.

[6] C. Hsieh, et al., Photoelectrochemical liftoff of patterned sapphire substrate for fabricating vertical light-emitting diode, IEEE Photon. Technol. Lett. 24 (2012) 1775.

[7] Rulian Wen, Xiaolong Hu, Quanbin Zhou, Ditao Chen, Hong Wang, Fabrication and characterization of high-efficiency green InGaN light-emitting diodes with metal-doped ITO layer, IEEE Trans. Electron. Dev. 65 (10) (2018) 4334.

[8] C.E. Lee, Y.C. Lee, H.C. Kuo, T.C. Lu, S.C. Wang, High brightness InGaN GaN flip-chip light-emitting diodes with triple-light scattering layers, IEEE Photon. Technol. Lett. 16 (8) (2008) 659.

[9] O.B. Shchekin, J.E. Epler, T.A. Trottier, T. Margalith, D.A. Steigerwald, M.O. Holcomb, P.S. Martin, M.R. Krames, High performance thin-film flip-chip InGaN-GaN light-emitting diodes, Appl. Phys. Lett. 89 (2006) 071109.

[10] Shengjun Zhou, Chenju Zheng, Jiajiang Lv, Yilin Gao, Ruiqing Wang, Sheng Liu, GaN-based flip-chip LEDs with highly reflective ITO/DBR p-type and via holebased n-type contacts for enhanced current spreading and light extraction, Optic Laser. Technol. 92 (2017) 95.

[11] Jiajiang Lv, Chenju Zheng, Quan Chen, Shengjun Zhou, Sheng Liu, High power InGaN/GaN flip-chip LEDs with via-hole-based two-level metallization electrodes, Phys. Status Solidi 213 (12) (2016) 3150.

[12] Shengjun Zhou, Xingtong Liu, Yilin Gao, Yingce Liu, Mengling Liu, Zongyuan Liu, Chengqun Gui, Sheng Liu, Numerical and experimental investigation of GaNbased flip-chip light-emitting diodes with highly reflective Ag/TiW and ITO/DBR Ohmic contacts, Optic Express 25 (22) (2017) 26615.

[13] Xingtong Liu, Shengjun Zhou, Yilin Gao, Hongpo Hu, Yingce Liu, Chengqun Gui, Sheng Liu, Numerical simulation and experimental investigation of GaN-based flip-chip LEDs and top-emitting LEDs, Appl. Optic. 56 (2017) 9502.

[14] Ismail Altuntas, Ilkay Demir, Ahmet Emre Kasapoğlu, Soheil Mobtakeri, Emre Gür, Sezai Elagoz, The effects of two-stage HT-GaN growth with different V/III ratios during 3D-2D transition, J. Phys. D Appl. Phys. 51 (2018) 035105.

[15] I. Gîrgel, A. Šatka, J. Priesol, P.-M. Coulon, E.D. Le Boulbar, T. Batten, D.W.E. Allsopp, P.A. Shields, Optical characterization of magnesium incorporation in pGaN layers for core-shell nanorod light-emitting diodes, J. Phys. D Appl. Phys. 51 (2018) 155103.

[16] Fatih Akyol, Digbijoy N. Nath, Emre Gür, Pil Sung Park, Siddharth Rajan, N-polar III-nitride green (540 nm) light emitting diode, Jpn. J. Appl. Phys. 50 (2011) 052101.

[17] Shengjun Zhou, Shu Yuan, Yingce Liu, L. Jay Guo, Sheng Liu, Han Ding, "Highly efficient and reliable high power LEDs with patterned sapphire substrate and strip-shaped distributed current blocking layer", Appl. Surf. Sci. 355 (2015) 1013. 